\title{
Comparison Maps for Relatively Free Resolutions ${ }^{\star}$
}

\author{
V. Álvarez, J.A. Armario, M.D. Frau, and P. Real \\ Depto. Matemática Aplicada I, E.T.S.I. Informática, Universidad de Sevilla, \\ Avda. Reina Mercedes, S.N. 41012 Sevilla (Spain) \\ \{valvarez, armario, mdfrau, real\}@us.es \\ http://www.us.es/gtocoma
}

\begin{abstract}
Let $\Lambda$ be a commutative ring, $A$ an augmented differential graded algebra over $\Lambda$ (briefly, DGA-algebra) and $X$ be a relatively free resolution of $\Lambda$ over $A$. The standard bar resolution of $\Lambda$ over $A$, denoted by $B(A)$, provides an example of a resolution of this kind. The comparison theorem gives inductive formulae $f: B(A) \rightarrow X$ and $g: X \rightarrow B(A)$ termed comparison maps. In case that $f g=1_{X}$ and $A$ is connected, we show that $X$ is endowed a $A_{\infty}$-tensor product structure. In case that $A$ is in addition commutative then $\left(X, \mu_{X}\right)$ is shown to be a commutative DGA-algebra with the product $\mu_{X}=f *(g \otimes g) \quad(*$ is the shuffle product in $B(A)$ ). Furthermore, $f$ and $g$ are algebra maps. We give an example in order to illustrate the main results of this paper.
\end{abstract}

\section{Introduction}

Calculations in homological algebra are commonly expressed in terms of resolutions. It is not unusual that these resolutions are embedded in the bar construction (or some other standard resolution) in a special way. When this occurs, they are said to split off of the standard resolution (see [14]).

A classic example is the Koszul resolution $K=A \otimes E_{\Lambda}\left[u_{1}, \ldots, u_{n}\right]$ related to the ideal $I=\left(x_{1}, \ldots, x_{n}\right)$ in the polynomial $\operatorname{ring} A=\Lambda\left[x_{1}, \ldots, x_{n}\right]$; as $A$ is an augmented algebra over $\Lambda$, the bar resolution $B(A)$ for $\Lambda$ over $A$ [5, 16] can be constructed. $K$ is also a resolution of $\Lambda$ over $A$ and by the comparison theorem [16, there is a chain homotopy equivalence $B(A) \leftarrow K$. In this case, an explicit contraction (special homotopy equivalence) $B(A) \Rightarrow K$ exists [17]. This contraction makes that the Koszul resolution splits off of the bar resolution. Using this contraction and some homological perturbation tools, perturbations of this resolution can be computed and the perturbed resolutions can be used to make complete effective calculations where previously only partial or indirect results were obtainable. This idea has been exploited in a series of papers by Lambe [12, 13, 14 and provides an algorithm for computing resolutions which split off of the bar construction. This algorithm has been extended to a more

\footnotetext{
^ This work was partially supported by the PAICYT research project FQM-296 from Junta de Andalucía (Spain).
} 
general context in [11]. We point out that the notion of contraction is essential in order to find effective algorithms in homological algebra using the set of techniques provided by homological perturbation theory, since the input data of our algorithm have to be codified in this form.

The well-known comparison theorem in homological algebra states that any two projective resolutions are chain homotopy equivalent. For relatively free resolutions $Y=A \otimes \bar{Y}$ and $X=A \otimes \bar{X}$ of $\Lambda$ over $A$ with explicit contracting homotopies, there are recursive procedures for obtaining explicit equivalences $f: Y \rightarrow X$ and $g: X \rightarrow Y$ where the explicit contracting homotopies play a principal rule. In addition, there are inductive procedures for obtaining explicit chain homotopies of $f g$ and the identity and with $g f$ and the identity. Generally, the maps defined in this way do not form a contraction. Here, assuming that $Y=B(A)$, we give a necessary and sufficient condition for determining when these maps form a contraction, which seems to be new.

In the special case that $A$ is connected, $Y$ is the bar resolution of $\Lambda$ over $A$, and the above maps form a contraction from $B(A)$ to $X$ (i.e., $X$ is a resolution which splits off of the bar resolution), we define a degree minus one map $\tau: \bar{X} \rightarrow A$ which is an $A_{\infty}$-twisting cochain, so that $\left(X, \tau,\left\{\Delta_{i}\right\}_{i \geq 0}\right)$ becomes an $A_{\infty}$-twisted tensor product where $\left\{\Delta_{i}\right\}_{i>0}$ is the $A_{\infty}$-coalgebra structure of $\bar{X}$ transferred from $\bar{B}(A)$ by means of 'tensor trick' [8] (see Algorithm 1). This provides an elegant codification of the differential of the complex $X$ in terms of the $A_{\infty}$ twisting cochain and the $A_{\infty}$-coalgebra structure of $\bar{X}$. Furthermore, assuming in addition that $A$ is commutative (but not necessarily connected) and that the contracting homotopy of $X$ is a quasi algebra homotopy (see [19]), we prove that the morphism $\mu_{X}=f *(g \otimes g)$ (* is the shuffle product in $\left.B(A)\right)$ endows $X$ a commutative algebra structure, for which $f$ and $g$ are algebra maps (see Theorem 7) and give a method for computing new resolutions taking advantage of this algebra structure (see Algorithm 2). In the example given in this paper, we compute a resolution $\widetilde{X}$ of $\mathbf{Z}_{p}$ over $\Gamma(w, 2 n)$ using an initial resolution which splits off of the standard resolution, $B(A) \Rightarrow X$, and perturbing this contraction. The contraction $B(A) \Rightarrow X$ has been computed by means of the comparison theorem. We prove that $X$ is a DGA-algebra as well as $\widetilde{X}$. A computational advantage is deduced from this fact, since it is only necessary to compute the perturbed differential on the generators of $\widetilde{X}$ as an algebra, better than on the whole set of generators as a module. This type of computational advantage is our main motivation for studying the algebra structures underlying the resolutions.

We organize the paper as follows. In section 2 we give the necessary definitions and notations for defining the comparison maps when $Y$ is the bar resolution and $X$ is a contractile relatively free resolution. We also give a necessary and sufficient condition for guaranteeing that a contraction arises. Section 3 is devoted to study the $A_{\infty}$-structure inherent in $X$, when $X$ is a relatively free resolution over a connected DGA-algebra $A$. In section 4 we analyse the multiplicative behaviour of the comparison maps, assuming that $A$ is a commutative DGA-algebra and that the contracting homotopy of $X$ is a quasi algebra homotopy. Finally, we give an example in order to illustrate the main results of the paper. 


\section{The Canonical Comparison Contraction - A Necessary and Sufficient Condition}

We will quickly review some basic notions of Homological Algebra. More details can be found in [16]. Let $\Lambda$ be a commutative ring with $1 \neq 0$, and $A$ an augmented differential graded algebra over $\Lambda$, briefly termed DGA-algebra. The differential, product, augmentation and coaugmentation of $A$ will be denoted respectively by $d_{A}, \mu_{A}, \epsilon_{A}$, and $\eta_{A}$. Nevertheless, sometimes, we will write them simply by $d, \mu, \epsilon$, and $\eta$ when no confusion can arise. In what follows, the Koszul sign conventions will be used. A morphism $\rho: A_{*} \rightarrow A_{*-1}$ is called derivation if it is compatible with the algebra structures on $A$. The degree of an element $a \in A$ is denoted by $|a|$. Let us recall that if $B$ is also a DGA-algebra, then $A \otimes B$ is canonically endowed an algebra structure by means of the morphism $\mu_{A \otimes B}=\left(\mu_{A} \otimes \mu_{B}\right)\left(1_{A} \otimes T \otimes 1_{B}\right)$, where $T(b \otimes a)=(-1)^{|b||a|} a \otimes b$. If the DGalgebra $A$ is connected, that is $A_{0}=\Lambda$ and $d_{1}: A_{1} \rightarrow A_{0}$ is zero, then there is a canonical augmentation $\epsilon_{A}=1_{\Lambda}: A_{0} \rightarrow \Lambda$.

Let $n$ be a positive integer. The exterior algebra with one generator $u$ in degree $2 n-1$, the polynomial algebra with one generator $v$ in degree $2 n$, and the divided power algebra with one "generator" $w$ in degree $2 n$ are denoted by $E(u, 2 n-1), P(v, 2 n)$, and $\Gamma(w, 2 n)$, respectively.

We need here the reduced bar construction $\bar{B}(A)$ of a DGA-algebra $A$ (see [16]). Recall that it is defined as the connected DGA-coalgebra, $\left.\bar{B}(A)=T^{c}(S(\bar{A}))\right)$, where $T^{c}()$ is the tensor coalgebra, $S()$ is the suspension functor, and $\bar{A}=$ Ker $\epsilon_{A}$ is the augmentation ideal of $A$. The element of $\bar{B}_{0}(A)$ corresponding to the identity element of $\Lambda$ is denoted by [ ] and the element $S \overline{a_{1}} \otimes \cdots \otimes S \overline{a_{n}}$ of $\bar{B}(A)$ is denoted by $\left[a_{1}|\cdots| a_{n}\right]$. The tensor and simplicial degrees of the element $\left[a_{1}|\cdots| a_{n}\right]$ are $\left|\left[a_{1}|\cdots| a_{n}\right]\right|_{t}=\sum\left|a_{i}\right|$ and $\left|\left[a_{1}|\cdots| a_{n}\right]\right|_{s}=n$, respectively; its total degree is the sum of its tensor and simplicial degree. The tensor and simplicial differential are defined by:

$$
d_{t}\left(\left[a_{1}|\cdots| a_{n}\right]\right)=-\sum_{i}(-1)^{e_{i-1}}\left[a_{1}|\cdots| d_{A}\left(a_{i}\right)|\cdots| a_{n}\right]
$$

and

$$
d_{s}\left(\left[a_{1}|\cdots| a_{n}\right]\right)=\sum_{i}(-1)^{e_{i}}\left[a_{1}|\cdots| \mu_{A}\left(a_{i} \otimes a_{i+1}\right)|\cdots| a_{n}\right]
$$

where

$$
e_{i}=i+\left|a_{1}\right|+\cdots+\left|a_{i}\right| \text {. }
$$

If the product of $A$ is commutative, a product $*$ (called shuffle product) can be defined on $\bar{B}(A)$. In this way, the reduced bar construction has a commutative Hopf algebra structure.

We will use here the structure of twisted tensor product. Let $A$ be a DGalgebra and $C$ a DG-coalgebra. It is well known that $\tau: C_{*} \rightarrow A_{*-1}$ is a twisting cochain if and only if $d^{\tau}=d_{A} \otimes 1+1 \otimes d_{C}+\tau \cap$ is a differential on $A \times C$ (see [4]), where the morphism $\tau \cap$ is defined by:

$$
\tau \cap=\left(\mu_{A} \otimes 1\right)(1 \otimes \tau \otimes 1)\left(1 \otimes \Delta_{C}\right) .
$$


The DG-module $\left(A \otimes C, d^{\tau}\right)$ is called the twisted tensor product (or TTP) of $A$ and $C$ along $\tau$. We will also use the notation of $A \otimes_{\tau} C$ for such DG-module.

A relatively free resolution of $\Lambda$ over $A$ is a pair $(X, \epsilon)$ where $X$ is a graded differential $A$-module of the form $X=A \otimes_{\Lambda} \bar{X}$ with $\bar{X}$ a DG- $\Lambda$-module and $\epsilon: X \rightarrow \Lambda$ a morphism of graded differential $A$-modules which is a weak equivalence, thereby, the homology of $X$ is zero except in degree 0 where it is $\Lambda$. We will call the complex $\left(\bar{X}, d_{\bar{X}}\right)$ the reduced complex, and it is always obtained in the form $\left(\bar{X}, d_{\bar{X}}\right)=\left(\Lambda \otimes_{A} X, 1_{\Lambda} \otimes_{A} d_{X}\right)$, by means of the the classical 'neglect' functor on the category of all $A$-modules to the category of all $\Lambda$-modules. It is standard terminology to call the elements of $\bar{X}$ reduced elements. Given a morphism $\psi: X \rightarrow Y$ the notation $\left.\psi\right|_{\bar{X}}(\bar{x})$ means $\psi(1 \otimes \bar{x})$ where 1 is the unit in $A$ and $\bar{x} \in \bar{X}$. We follow these conventions throughout the paper.

A resolution $\epsilon:(X, d) \rightarrow \Lambda$ is called contractile if there exists a 'contracting homotopy', i.e., a family of $\Lambda$-module morphisms, $h_{-1}: \Lambda \rightarrow X_{0}, h_{n}: X_{n} \rightarrow X_{n+1}$, such that $1=d_{n+1} h_{n}+h_{n-1} d_{n}, \forall n \geq 0$, where $d_{0}=\epsilon$ and $h_{-1}=\eta$. Besides, it may always be assumed to hold that $h^{2}=0$ (see [3]).

Throughout this paper, $(X, h, d)$ will denote a contractile relatively free resolution $(X, d)$ with contracting homotopy $h$.

An important example of relatively free and contractile resolution of $\Lambda$ over $A$ is the bar resolution $(B(A), s, d)$ (or $B(A))$ [16,13. More specifically, $B(A)$ is the twisted tensor product $A \otimes_{\theta} \bar{B}(A)$, where the twisting cochain $\theta$ is given by

$$
\theta\left(\left[a_{1}|\cdots| a_{n}\right]\right)= \begin{cases}a_{1} & n=1 \\ 0 & \text { otherwise }\end{cases}
$$

where the weak equivalence $\epsilon_{B(A)}: B(A) \rightarrow \Lambda$ is the canonical augmentation of $B(A)$ (in fact, it is a homotopy equivalence) and the contracting homotopy $s$ is given by

$$
s: B(A) \rightarrow B(A) \quad \text { where } \quad s\left(a \otimes\left[a_{1}|\cdots| a_{n}\right]\right)=\left[a\left|a_{1}\right| \cdots \mid a_{n}\right]
$$

From now on, we will use $s$ for denoting the above homotopy.

A contraction (see [6, 9]) is a data set $c:\{N, M, f, g, \phi\}$ where $f: N \rightarrow M$ and $g: M \rightarrow N$ are morphisms of DG-modules (called, respectively, projection and inclusion) and $\phi: N \rightarrow N$ is a morphism of graded modules of degree +1 (called homotopy operator). These data are required to satisfy the rules: (c1) $f g=1_{M},(\mathbf{c 2}) \phi d_{N}+d_{N} \phi+g f=1_{N}$ (c3) $\phi \phi=0,(\mathbf{c} 4) \phi g=0$ and (c5) $f \phi=0$. These three last are called side conditions [15]. In fact, these may always be assumed to hold, since the homotopy $\phi$ can be altered to satisfy these conditions 7, 14. We will also denote a contraction $c$ by $(f, g, \phi): N \Rightarrow M$.

For instance, the bar resolution $B(A)$ of a DG-algebra $A$ gives the following contraction:

$$
C_{B(A)}:\left\{B(A), \Lambda, \epsilon_{B(A)}, \eta_{B(A)}, s\right\}
$$

where $\eta_{B(A)}: A \rightarrow B(A)$ is the canonical coaugmentation of $B(A)$. 
By the comparison theorem for resolutions [16, given any relatively free resolution $X=\left(A \otimes \bar{X}, d_{X}\right) \stackrel{\epsilon}{\longrightarrow} \Lambda$ of $\Lambda$ over $A$, there is an $A$-lineal morphism ( $a$ comparison map) $g: X \rightarrow B(A)$ inductively defined:

$$
\left.g_{0}\right|_{\bar{x}_{0}}=\left.\eta_{B(A)} \epsilon_{X}\right|_{\bar{x}_{0}},\left.\quad g_{n+1}\right|_{\bar{x}_{n+1}}=\left.s g_{n} d_{n+1}\right|_{\bar{x}_{n+1}} .
$$

This map is a homotopy equivalence between $B(A)$ and $X$. Moreover, if the resolution $X$ is contractile with contracting homotopy $t,(X, t, d)$, then comparison theorem provides an analogous inductive definition for the $A$-lineal morphism $f: B(A) \rightarrow X:$

$$
\left.f_{0}\right|_{\bar{B}(A)_{0}}=\left.\eta_{X} \epsilon_{B(A)}\right|_{\bar{B}(A)_{0}},\left.\quad f_{n+1}\right|_{\bar{B}(A)_{n+1}}=\left.t f_{n} d_{n+1}\right|_{\bar{B}(A)_{n+1}} .
$$

Both of the compositions $f g, g f$ of theses comparison maps are homotopic to the corresponding identity maps. Inductive formulae for the associated homotopies are also available:

$$
\phi: B(A)_{*} \rightarrow B(A)_{*+1}
$$

defined on reduced elements and then extended $A$-linearly,

$$
\left.\phi\right|_{\bar{B}(A)}=\left.(-s g f-s \phi d)\right|_{\bar{B}(A)},
$$

and

$$
\kappa: X_{*} \rightarrow X_{*+1}
$$

where

$$
\kappa=t(1-g f) .
$$

Let us observe that in general $\kappa$ is not $A$-lineal. These formulae are crucial for the work in 13,14]. Let us observe that the morphisms $g$ and $\phi$ satisfy

$$
g(\bar{X}) \subseteq \bar{B}(A), \quad \phi(\bar{B}(A)) \subseteq \bar{B}(A),
$$

but $f$ and $\kappa$ do not satisfy the analogous condition.

Generally, $f g$ is different to $1_{X}$, but sometimes a contraction arises, which we call 'the canonical comparison contraction'. A necessary condition for guaranteeing that $f g=1_{X}$ is given in [11. We next give a necessary and sufficient condition for this purpose.

Theorem 1. The data set $\mathcal{C}:\{B(A),(X, t, d), f, g, \phi\}$ is a contraction if and only if $\left.d t\right|_{\bar{x}}=0$.

Proof. First we assume that $\left.d t\right|_{\bar{X}}=0$. Taking into account that $t$ is a contracting homotopy of $X$ to $\Lambda$, it holds that

$$
\left.1\right|_{\bar{X}}=\left.(d t+t d)\right|_{\bar{X}}=\left.t d\right|_{\bar{X}}
$$

Now we will show that $f g=1_{X}$, the proof is by induction. We have $f_{0} g_{0}=1$ by construction and for $n>0$, on reduced elements,

$$
f_{n} g_{n}=t f_{n-1} d_{n} g_{n}=t f_{n-1} g_{n-1} d_{n}=t d_{n}=1
$$


where the first and third equality comes from (7) and induction hypothesis on $t$, respectively. By $A$-linearity the proof is extended to elements of $X$. It is readily checked that the side conditions hold.

Reciprocally, now, let us assume that $f g=1_{X}$. Working in a similar way as above, we have, on reduced elements for $n \geq 0$,

$$
1=f_{n} g_{n}=t f_{n-1} d_{n} g_{n}=t f_{n-1} g_{n-1} d_{n}=t d_{n}
$$

Hence, $\left.t d\right|_{\bar{X}}=\left.1\right|_{\bar{X}}$ and $\left.d t\right|_{\bar{X}}=0$.

Remark 1. For the remainder of this paper, we will assume that $\mathcal{C}$ is a contraction. In this situation, $\mathcal{C}$ is called the canonical comparison contraction for $(X, t, d)$.

A resolution $X$ splits off of the bar construction (see [14]) if there is a contraction (called comparison contraction) from $B(A)$ to $X$. Note that this contraction can be different from the canonical one.

With this definition at hand, we can state the following proposition:

Proposition 1. Let $(X, t, d)$ be a contractile relatively free resolution. If $\left.d t\right|_{\bar{X}}=$ 0 , then $X$ splits off of the bar construction.

In the sequel proposition we analyze the contracting homotopy $t$ of $(X, t, d)$.

Proposition 2. Let $(X, t, d)$ be a contractile relatively free resolution which splits off of the bar construction by means of the canonical comparison contraction. Then $t=f s g$.

Proof. First, due to the fact that $s: B(A) \rightarrow \bar{B}(A)$, we can use the inductive definition of $f$ in this composition $f s$, thus

$$
f s g=(t f d) s g
$$

since $s d+d s=1$,

$$
t f(d s) g=t f(1-s d) g=t f g-t f s d g=t f g .
$$

The last identity results from the fact that $t f s=t(t f d) s=0$ (because $t^{2}=0$ ). Finally,

$$
t f g=t
$$

since $f g=1_{X}$.

Remark 2. If we consider $\epsilon_{Y}:\left(Y, t_{Y}, d\right) \rightarrow \Lambda$ any contractile relatively free resolution of $\Lambda$ over $A$, instead of $B(A)$, the comparison theorem for resolutions provides similar formulae (comparison maps):

$$
f: Y \rightarrow X, \quad g: X \rightarrow Y, \quad \phi: Y_{*} \rightarrow Y_{*+1}, \quad \kappa: X_{*} \rightarrow X_{*+1}
$$

where under the hypothesis that $t_{Y}(Y) \subset \bar{Y}$, it is possible to get the result analogous to Theorem 1 . 


\section{Differential Structures in the Comparison of Resolutions}

Up to now little has been said about the nature of the differential $d_{X}$ in $X$ for an arbitrary contractile relatively free resolution with contracting homotopy $t$. Here we show that $d_{X}$ can be rewritten in terms of an $A_{\infty}$-twisting cochain $\tau: \bar{X} \rightarrow A$ and the $A_{\infty}$-coalgebra structure of $\bar{X}$ transferred from the coalgebra structure of $\bar{B}(A)$. To this end, we prove two previous results (Theorems 4 and 5 ) which claim that working with resolutions (à la Cartan) is equivalent to work with reduced complexes (à la Eilenberg-Mac Lane) from a homogical point of view. We describe a method for passing from one way to the other.

Now, we recall the concept of a perturbation datum. Let $N$ be a graded module and let $f: N \rightarrow N$ be a morphism of graded modules. The morphism $f$ is pointwise nilpotent if for all $x \in N(x \neq 0)$, a positive integer $n$ exists (in general, the number $n$ depends on the element $x$ ) such that $f^{n}(x)=0$. A perturbation of a DG-module $N$ is a morphism of graded modules $\delta: N \rightarrow N$ of degree -1 , such that $\left(d_{N}+\delta\right)^{2}=0$ and $\delta_{1}=0$. A perturbation datum of the contraction $c:\{N, M, f, g, \phi\}$ is a perturbation $\delta$ of the DGA-module $N$ verifying that the composition $\phi \delta$ is pointwise nilpotent.

We now introduce the main tool in Homological Perturbation Theory: the Basic Perturbation Lemma ([4, 7, 8, 3, 19]).

\section{Theorem 2. (BPL)}

Let $c:\{N, M, f, g, \phi\}$ be a contraction and $\delta: N \rightarrow N$ be a perturbation datum of c. Then, a new contraction

$$
c_{\delta}:\left\{\left(N, d_{N}+\delta\right),\left(M, d_{M}+d_{\delta}\right), f_{\delta}, g_{\delta}, \phi_{\delta}\right\}
$$

is defined by the formulas: $d_{\delta}=f \delta \Sigma_{c}^{\delta} g ; f_{\delta}=f\left(1-\delta \Sigma_{c}^{\delta} \phi\right) ; g_{\delta}=\Sigma_{c}^{\delta} g ; \phi_{\delta}=\Sigma_{c}^{\delta} \phi$; where

$$
\Sigma_{c}^{\delta}=\sum_{i \geq 0}(-1)^{i}(\phi \delta)^{i}=1-\phi \delta+\phi \delta \phi \delta-\cdots+(-1)^{i}(\phi \delta)^{i}+\cdots
$$

Let us note that $\Sigma_{c}^{\delta}(x)$ is a finite sum for each $x \in N$ because of the pointwise nilpotency of the composition $\phi \delta$. Moreover, it is obvious that the morphism $d_{\delta}$ is a perturbation of the DG-module $\left(M, d_{M}\right)$.

The transference of the algebra structure up to homology equivalence has been considered in [8, 9, 19. Next, we review several notions.

Definition 1. [19] Let $A$ and $A^{\prime}$ be two DG-algebras and $c:\left\{A, A^{\prime}, f, g, \phi\right\}$ be a contraction. The projection $f$ is a quasi algebra projection if the following conditions hold:

$$
f \mu_{A}(\phi \otimes \phi)=0, \quad f \mu_{A}(\phi \otimes g)=0, \quad f \mu_{A}(g \otimes \phi)=0 .
$$

The homotopy operator $\phi$ is a a quasi algebra homotopy if the following conditions hold:

$$
\phi \mu_{A}(\phi \otimes \phi)=0, \quad \phi \mu_{A}(\phi \otimes g)=0, \quad \phi \mu_{A}(g \otimes \phi)=0 .
$$


Definition 2. [8] Let $A$ and $A^{\prime}$ be two DG-algebras and $c:\left\{A, A^{\prime}, f, g, \phi\right\}$ be a contraction. The homotopy operator $\phi$ is said to be an algebra homotopy if

$$
\phi \mu_{A}=\mu_{A}\left(1_{A} \otimes \phi+\phi \otimes g f\right) .
$$

Definition 3. [19] Let $A$ and $A^{\prime}$ be two DGA-algebras and $r:\left\{A, A^{\prime}, f, g, \phi\right\}$ a contraction. We say that $r$ is

- a semi-full algebra contraction if $f$ is a quasi algebra projection, $g$ is a morphism of DGA-algebras and $\phi$ is a quasi algebra homotopy.

- an almost-full algebra contraction if $f$ and $g$ are morphisms of DGA-algebras and $\phi$ is a quasi algebra homotopy.

- a full algebra contraction if $f$ and $g$ are morphisms of DGA-algebras and $\phi$ is an algebra homotopy.

Obviously, full and almost-full algebra contractions are, in particular, semi-full algebra contractions. It is not difficult to prove that both sets of semi-full and almost-full algebra contractions are closed by composition and tensor product of contractions.

If $A$ is a commutative DGA-algebra, the contraction (3) is an example of an almost-full algebra contraction.

Definition 4. [7] Let $A$ and $A^{\prime}$ be two DG-algebras and $c:\left\{A, A^{\prime}, f, g, \phi\right\}$ a contraction. An algebra perturbation datum $\delta$ of $c$ is a perturbation datum of this contraction which is also a derivation.

The following result tells us that the set of semi-full algebra contractions is closed by homological perturbation. This theorem is used in the proof of some theorems of this paper.

Theorem 3 (SF-APL). ([19])

Taking as data a semi-full algebra contraction $r$ and an algebra perturbation datum $\delta$ of $r$, the perturbed contraction $r_{\delta}$ is an algebra contraction of the same type, where the product on $A^{\prime}{ }_{\delta}$ is the original product $\mu_{A^{\prime}}$.

\subsection{From Resolutions to Reduced Complexes}

Throughout this subsection, $A$ will denote a connected DGA-algebra.

The goal of this subsection is to establish a contraction from $\bar{B}(A)$ to $\bar{X}$ ('reduced complexes') by means of the canonical comparison contraction between the contractile relatively free resolutions $B(A)$ and $(X, t, d)$ :

$$
\mathcal{C}:\{B(A),(X, t, d), f, g, \phi\} .
$$

To this end, we will apply the classical 'neglect' functor on the category of all $A$ modules to the category of all $\Lambda$-modules, $\Lambda \otimes_{A}-$ and $1 \otimes_{A}-$, on the complexes and morphisms involved in the above contraction, respectively. 
The following properties will play an important role in what follows.

$$
\begin{aligned}
& \text { 1. } X \cong\left(\operatorname{Ker} \epsilon_{A} \otimes \bar{X}\right) \oplus(\Lambda \otimes \bar{X}) . \\
& \text { 2. } \Lambda \otimes_{A} X \cong \Lambda \otimes \bar{X} \text { and } 1 \otimes_{A} d_{X}=\left(\epsilon_{A} \otimes 1_{\bar{X}}\right) d_{X} \text {. } \\
& \text { 3. } 1 \otimes_{A} g=\left.g\right|_{\bar{X}}, \quad 1 \otimes_{A} \phi=\left.\phi\right|_{\bar{B}(A)} \text {. } \\
& \text { 4. } 1 \otimes_{A} f=\left(\epsilon_{A} \otimes 1_{\bar{X}}\right) f . \\
& \text { 5. } f(\bar{b})=0 \Rightarrow\left(1 \otimes_{A} f\right)(\bar{b})=0 . \\
& \text { 6. } d_{X}(\bar{x})=0 \Rightarrow\left(1 \otimes_{A} d_{X}\right)(\bar{x})=0 .
\end{aligned}
$$

Properties 1, 2, and 4 are deduced from the meaning of tensoring by $A$. Since $g$ and $\phi$ are $A$-lineal and satisfy (7), the third is followed. Properties 5 and 6 are consequences of 1 and 2 .

By property 3 we have that $1 \otimes_{A} g$ and $1 \otimes_{A} \phi$ are DGA-module morphisms. In spite of the fact that $f$ does not satisfy (7), we will prove that $1 \otimes_{A} f$ is a morphism of DGA-modules as well.

Firstly, note that

$$
\left(1 \otimes_{A} f\right)\left(1 \otimes_{A} d\right)=\left(\epsilon_{A} \otimes 1\right) f\left(\epsilon_{A} \otimes 1\right) d=\left(\epsilon_{A} \otimes 1\right) f d=\left(\epsilon_{A} \otimes 1\right) d f
$$

here we have used that $f$ is $A$-lineal and a DGA-module morphism as well.

On the other hand,

$$
\left(1 \otimes_{A} d\right)\left(1 \otimes_{A} f\right)=\left(\epsilon_{A} \otimes 1\right) d\left(\epsilon_{A} \otimes 1\right) f
$$

Now, taking into account that the differential $d$ of an $A$-module $X$ satisfies

$$
d(a \otimes \bar{x})=d_{A}(a) \otimes \bar{x}+(-1)^{|a|} a \otimes d(\bar{x})
$$

it is clear that if $a \otimes \bar{x} \in \operatorname{Ker} A \otimes \bar{X}$ then $d(a \otimes \bar{x}) \in \operatorname{Ker} A \otimes \bar{X}$. Thereby,

$$
\left(\epsilon_{A} \otimes 1\right) d f=\left(\epsilon_{A} \otimes 1\right) d\left(\epsilon_{A} \otimes 1\right) f .
$$

The properties required for this data set

$$
\left\{\bar{B}(A), \bar{X}, 1 \otimes_{A} f, 1 \otimes_{A} g, 1 \otimes_{A} \phi\right\}
$$

in order to be a contraction are inherited from $\mathcal{C}$ in a straightforward manner. Therefore, we can state:

Theorem 4. The data set $1 \otimes_{A} \mathcal{C}:\left\{\bar{B}(A), \bar{X}, 1 \otimes_{A} f, 1 \otimes_{A} g, 1 \otimes_{A} \phi\right\}$ is a contraction. 


\subsection{From Reduced Complexes to Resolutions}

Taking as input a contraction from the reduced bar construction of a connected DGA-algebra $A, \bar{B}(A)$, to a free DGA-module $\bar{X}$, we describe [2] a method for obtaining resolutions which split off of the bar construction. This process plays an important role in the main result of this section.

Proposition 3. [2] Let $A$ be a connected DGA-algebra. Given a contraction c from $\left(\bar{B}(A), d_{\bar{B}(A)}\right)$ to a DGA-module $(\bar{X}, \bar{d})$, in which the homotopy operator increases the simplicial degree by one, there is a comparison contraction from the bar resolution $B(A)$ to the resolution $X$, where the underlying module in $X$ is just the $A \otimes \bar{X}$ and the differential structure is done via perturbation of $c$.

Theorem 5. Assuming that $(X, t, d)$ is a contractile relatively free resolution which splits off of the bar construction of a connected DGA-algebra A, under the canonical comparison contraction, then $\theta \cap$ is a perturbation datum for

$C:\left\{A \otimes\left(\Lambda \otimes_{A} B(A)\right), A \otimes\left(\Lambda \otimes_{A} X\right), 1 \otimes\left(1 \otimes_{A} f\right), 1 \otimes\left(1 \otimes_{A} g\right), 1 \otimes\left(1 \otimes_{A} \phi\right)\right\}$

and the perturbed contraction $C_{\theta \cap}$ coincides with the canonical comparison contraction.

Proof. Theorem 4 of subsection 3.1 states that from the canonical comparison contraction $\mathcal{C}:\{B(A), X, f, g, \phi\}$ it is possible to establish a contraction between the reduced complexes

$$
\mathcal{C}^{\otimes_{A}}:\left\{\Lambda \otimes_{A} B(A), \Lambda \otimes_{A} X, 1 \otimes_{A} f, 1 \otimes_{A} g, 1 \otimes_{A} \phi\right\} .
$$

Proposition $[$ states that if $A$ is connected then $\theta \cap$ is a perturbation datum of the contraction

$1 \otimes \mathcal{C}^{\otimes_{A}}:\left\{A \otimes\left(\Lambda \otimes_{A} B(A)\right), A \otimes\left(\Lambda \otimes_{A} X\right), 1 \otimes\left(1 \otimes_{A} f\right), 1 \otimes\left(1 \otimes_{A} g\right), 1 \otimes\left(1 \otimes_{A} \phi\right)\right\}$

Now, we prove that the perturbed contraction $\left(1 \otimes \mathcal{C}^{\otimes_{A}}\right)_{\theta \cap}$ coincides with $\mathcal{C}$. To this end, it suffices to show that the formulae

$$
\begin{aligned}
& f_{\theta \cap}=1 \otimes \bar{f}-(1 \otimes \bar{f}) \theta \cap(1 \otimes \bar{\phi})+(1 \otimes \bar{f}) \theta \cap(1 \otimes \bar{\phi}) \theta \cap(1 \otimes \bar{\phi})-\ldots \\
& g_{\theta \cap}=1 \otimes \bar{g}-(1 \otimes \bar{\phi}) \theta \cap(1 \otimes \bar{g})+(1 \otimes \bar{\phi}) \theta \cap(1 \otimes \bar{\phi}) \theta \cap(1 \otimes \bar{g})-\cdots \\
& \phi_{\theta \cap}=1 \otimes \bar{\phi}-(1 \otimes \bar{\phi}) \theta \cap(1 \otimes \bar{\phi})+(1 \otimes \bar{\phi}) \theta \cap(1 \otimes \bar{\phi}) \theta \cap(1 \otimes \bar{\phi})-\cdots \\
& d_{\theta \cap}=D \quad+(1 \otimes \bar{f}) \theta \cap(1 \otimes \bar{g})-(1 \otimes \bar{f}) \theta \cap(1 \otimes \bar{\phi}) \theta \cap(1 \otimes \bar{g})+\cdots
\end{aligned}
$$

coming from the BPL are the morphisms integrating $\mathcal{C}$, where $D$ denotes the usual differential over $A \otimes\left(\Lambda \otimes_{A} X\right)$, and $\bar{h}$ denotes $1 \otimes_{A} h$.

Let us recall that $1 \otimes_{A} g=\left.g\right|_{\bar{X}}, 1 \otimes_{A} \phi=\left.\phi\right|_{\bar{B}(A)}$. Furthermore,

$(\theta \cap) s\left(a \otimes\left[a_{1}|\ldots| a_{n}\right]\right)=\left\{\begin{array}{cl}a \otimes\left[a_{1}|\ldots| a_{n}\right] & \text { if } a \otimes\left[a_{1}|\ldots| a_{n}\right] \in \operatorname{Ker} \epsilon_{A} \otimes \bar{B}(A) \\ 0 & \text { elsewhere }\end{array}\right.$ 
Taking into account the above identities and the inductive definitions of $f, g$, and $\phi$ it follows that

$(\theta \cap)(1 \otimes \bar{\phi})=-\left.(\theta \cap)(1 \otimes s \phi d+1 \otimes s g f)\right|_{\bar{B}}=-\left.\left(\mu_{A} \otimes 1\right)\right|_{A \otimes K \operatorname{Ker} \epsilon_{A} \otimes \bar{B}}\left(\left.1 \otimes(\phi d+g f)\right|_{\bar{B}}\right)$

and,

$$
(\theta \cap)(1 \otimes \bar{g})=(\theta \cap)\left(\left.1 \otimes s g d\right|_{\bar{B}}\right)=\left.\left(\mu_{A} \otimes 1\right)\right|_{A \otimes K e r \epsilon_{A} \otimes \bar{B}}\left(\left.1 \otimes g d\right|_{\bar{B}}\right) .
$$

In view of the previous identities and using that $\phi \phi=0, \phi g=0$ and (7), we have that

$$
\begin{gathered}
(1 \otimes \bar{\phi}) \theta \cap(1 \otimes \bar{\phi})=-\left.(1 \otimes \bar{\phi})\left(\mu_{A} \otimes 1\right)\right|_{A \otimes K \operatorname{Ker} \epsilon_{A} \otimes \bar{B}}\left(\left.1 \otimes(\phi d+g f)\right|_{\bar{B}}\right) \\
=-\left.\left(\mu_{A} \otimes 1\right)\right|_{A \otimes K \operatorname{Ker} \epsilon_{A} \otimes \bar{B}}\left(\left.1 \otimes \phi(\phi d+g f)\right|_{\bar{B}}\right)=0 .
\end{gathered}
$$

Furthermore,

$$
\begin{gathered}
(1 \otimes \bar{\phi}) \theta \cap(1 \otimes \bar{g})=\left.(1 \otimes \bar{\phi})\left(\mu_{A} \otimes 1\right)\right|_{A \otimes K \operatorname{Ker} \epsilon_{A} \otimes \bar{B}}\left(\left.1 \otimes g d\right|_{\bar{B}}\right) \\
=\left.\left(\mu_{A} \otimes 1\right)\right|_{A \otimes K e r \epsilon_{A} \otimes \bar{B}}\left(\left.1 \otimes \phi g d\right|_{\bar{B}}\right)=0 .
\end{gathered}
$$

Thus, the formulae (9) may now be rewritten as

$$
\begin{gathered}
f_{\theta \cap}=1 \otimes \bar{f}-(1 \otimes \bar{f}) \theta \cap(1 \otimes \bar{\phi}), \quad g_{\theta \cap}=1 \otimes \bar{g}, \\
\phi_{\theta \cap}=1 \otimes \bar{\phi}, \quad d_{\theta \cap}=D+(1 \otimes \bar{f}) \theta \cap(1 \otimes \bar{g}) .
\end{gathered}
$$

Obviously, $g_{\theta \cap}$ and $\phi_{\theta \cap}$ are the $A$-lineal extensions of $\bar{g}$ and $\bar{\phi}$, hence $g_{\theta \cap}=g$ and $\phi_{\theta \cap}=\phi$. Working out the second summand of $f_{\theta \cap}$, we have that

$$
\begin{gathered}
-(1 \otimes \bar{f}) \theta \cap(1 \otimes \bar{\phi})=\left.\left(\mu_{A} \otimes \bar{f}\right)\right|_{A \otimes K e r \epsilon_{A} \otimes \bar{B}}\left(\left.1 \otimes(\phi d+g f)\right|_{\bar{B}}\right) \\
=\left.\left(\mu_{A} \otimes \bar{f}\right)\right|_{A \otimes K e r \epsilon_{A} \otimes \bar{B}}\left(\left.1 \otimes \phi d\right|_{\bar{B}}\right)+\left.\left(\mu_{A} \otimes \bar{f}\right)\right|_{A \otimes K e r \epsilon_{A} \otimes \bar{B}}\left(\left.1 \otimes g f\right|_{\bar{B}}\right) .
\end{gathered}
$$

By property 5 of (8) the first term is zero, since $f \phi=0$. The second one, acting over an element $\eta_{A}(1) \otimes\left[a_{1}|\ldots| a_{n}\right]$ coincides with the summands of $f\left(\left[a_{1}|\ldots| a_{n}\right]\right)$ which becomes zero when the functor $1 \otimes_{A}-$ is applied over it, since $f g f=f$. Hence, $f_{\theta \cap}=f$.

In a similar way, it is proved that $d_{\theta \cap}=d$.

\section{3 $A_{\infty}$-Structures and HPT}

The notion of an $A_{\infty}$-(co)algebra was introduced by J. Stasheff [20], which is "roughly speaking" a differential graded (co)algebra in which the (co)associative law holds up to homotopy. Here we deal with the category of $A_{\infty}$-coalgebras. Given a DG-module $\left(M, \Delta_{1}\right)$ and a sequence of maps $\left\{\Delta_{i} \in \operatorname{Hom}^{i-2}\left(M, M^{\otimes i}\right)\right\}_{i \geq 1}$, $\left(M, \Delta_{i}\right)_{n \geq 1}$ is called an $A_{\infty}$-coalgebra if the relation

$$
\sum_{n=1}^{i} \sum_{k=0}^{i-n}(-1)^{n+k+n k}\left(1^{i-n-k} \otimes \Delta_{n} \otimes 1^{k}\right) \Delta_{i-n+1}=0
$$

holds for each $i \geq 1$. 
The problem of transferring (co)algebra structures up to contraction has been largely considered in the literature. Here we will need the following results:

Lemma 1. [8] Assuming that $C$ is a coalgebra, $M$ a DGA-module and $r:\{C, M, f, g, \phi\}$ a contraction, and using the tensor trick (see [8, 9, 10]), then $M$ becomes an $A_{\infty}$-coalgebra.

Moreover, the maps integrating the $A_{\infty}$-coalgebra $\left(M, \Delta_{i}\right)_{i \geq 1}$ are shown in [1] to be explicitly

$$
\Delta_{i}=(-1)^{\frac{(i-1)(i-2)}{2}} f^{\otimes i_{\circ}}
$$

$$
\circ\left[\sum_{k_{2}=1}^{2} \sum_{k_{3}=1}^{k_{2}+1} \cdots \sum_{k_{i-1}=1}^{k_{i-2}+1} \prod_{j=2}^{i-1}(-1)^{k_{j}}\left(1^{\otimes k_{j}-1} \otimes \Delta_{C} \phi \otimes 1^{\otimes j-k_{j}}\right)\right] \Delta_{C} g
$$

where $\prod_{j=2}^{i-1} h_{j}$ denotes the composition $h_{i-1} \circ \cdots \circ h_{2}$.

Given a DGA-algebra $A$ and an $A_{\infty}$-coalgebra $C$, an $A_{\infty}$-twisting cochain (or $A_{\infty}$-TTP) $\tau: C \rightarrow A$ is a DG-module morphism of degree -1 , such that satisfies the following identity

$$
d \tau+\sum_{i=1}^{\infty} \mu^{(i)} \tau^{\otimes i} \Delta_{i}=0
$$

where $\mu^{(1)}=1, \mu^{(2)}=\mu$, and in general $\mu^{(k)}=\mu\left(1 \otimes \mu^{(k-1)}\right)$. Analogously to twisting cochain, $\tau: C \rightarrow A$ is an $A_{\infty}$-twisting cochain [18] if and only if $d_{\tau}=d \otimes 1+\sum_{i=1}^{\infty}\left(\mu^{(i)} \otimes 1\right)\left(1 \otimes \tau^{\otimes i-1} \otimes 1\right)\left(1 \otimes \Delta_{i}\right)$ is a differential on $A \otimes C$, which, together with this differential, is denoted by $A \otimes_{\tau} C$ and is referred to as the $A_{\infty}$-twisted tensor product (along $\tau$ ).

Theorem 6. [1] Let $t: C \rightarrow A$ be a twisting cochain and $c(f, g, \phi): C \Rightarrow C^{\prime}$ be a contraction such that $c$ induces on $C^{\prime}$ an $A_{\infty}$-coalgebra structure (see Lemma 1). Additionally, assume that $t \phi=0$ and $(1 \otimes \phi) t \cap$ is pointwise nilpotent. There is a contraction

$$
A \otimes_{t} C \Rightarrow A \otimes_{\bar{t}} C^{\prime}
$$

where $\bar{t}=\operatorname{tg}$ is an $A_{\infty}$-twisting cochain and $A \otimes_{\bar{t}} C^{\prime}$ is an $A_{\infty}$-twisted tensor product.

Remark 3. The hypotheses of Theorem 6 are satisfied when $C$ is a simply connected DGA-coalgebra. For instance, $\bar{B}(A)$ is a simply connected DGA-coalgebra when $A$ is a connected DGA-algebra.

The following result is a straightforward consequence of Theorem 6, and it is a main result of this paper. 
Algorithm 1. Computing the $A_{\infty}$-twisting tensor product structure

INPUT: A contractile relatively free resolution $(X, t, d)$ of $\Lambda$ over a connected DG-algebra $A$ where $\left.d \circ t\right|_{\bar{X}}=0$.

Step 1. Form the canonical comparison contraction $(f, g, \phi): B(A) \Rightarrow X$ using the formulas (5), (4) and (6) .

Step 2. Form the contraction $\left(1 \otimes_{A} f, 1 \otimes_{A} g, 1 \otimes_{A} \phi\right): \bar{B}(A) \Rightarrow \bar{X}$ as in Theorem 4 .

Output: The map $\tau=\left.\theta g\right|_{\bar{x}}$ which is an $A_{\infty}$-twisting cochain and the maps $\Delta_{i}$ (given by formula (10) using the morphisms of the contraction of Step 2) integrating the $A_{\infty}$-coalgebra structure $\left(\bar{X}, \Delta_{i}\right)_{i \geq 1}$.

Correctness: Let us emphasize that $1 \otimes_{A} g=\left.g\right|_{\bar{X}}$ and $1 \otimes_{A} \phi=\left.\phi\right|_{\bar{B}(A)}$ since $g$ and $\phi$ are $A$-lineal and $g(\bar{X}) \subseteq \bar{B}(A)$, and $\phi(\bar{B}(A)) \subseteq \bar{B}(A)$. An explicit formula for $\phi$ is given in 13, 14, which increases the simplicial degree in $\bar{B}(A)$ by one and $\phi_{0}=0$. Therefore, $\left.\theta \phi\right|_{\bar{B}(A)}=0$ since $\theta: \bar{B}(A) \rightarrow A$ is the universal twisting cochain. Now, applying Theorem [6 we have the following $A_{\infty}$-twisting cochain

$$
\tau=\left.\theta g\right|_{\bar{X}}: \bar{X} \rightarrow A
$$

The second step is to construct the tensor product contraction

$$
A \otimes \bar{B}(A) \Rightarrow A \otimes \bar{X}
$$

and to use the Basic Perturbation Lemma with $\theta \cap$ as the perturbation datum (see Theorem 5). Then, it is straightforward to check that $\left(\left.1 \otimes \phi\right|_{\bar{B}(A)}\right) \theta \cap$ is pointwise nilpotent. So we obtain the new contraction,

$$
A \otimes_{\theta} \bar{B}(A) \Rightarrow\left(A \otimes \bar{X}, d_{\theta \cap}\right)
$$

Now, using Theorem [6, we have that

$$
\left(A \otimes \bar{X}, d_{\theta \cap}\right)=A \otimes_{\tau} \bar{X}
$$

where $A \otimes_{\tau} \bar{X}$ is an $A_{\infty}$-twisted tensor product.

In the proof of the last identity, we use the special properties of the morphisms which take part in the canonical comparison contraction.

\section{Algebra Structures in the Comparison of Resolutions}

If $A$ is a commutative DGA-algebra, it is well known that it is possible to define a commutative product $*$ on $\bar{B}(A)$ called shuffle product. Furthermore, $B(A)$ has canonically associated a commutative algebra structure by means of the morphism $\mu_{B(A)}=\left(\mu_{A} \otimes *\right)\left(1_{A} \otimes T \otimes 1_{\bar{B}(A)}\right)$, where $T(\bar{b} \otimes a)=(-1)^{|\bar{b}||a|} a \otimes \bar{b}$. 
Throughout this section, we assume that $A$ is a commutative DGA-algebra, $(X, t, d)$ is a contractile relatively free resolution with $t$ as contracting homotopy which is a quasi algebra homotopy, and there exists $\mathcal{C}:\{B(A), X, f, g, \phi\}$ the canonical comparison contraction.

Before giving the main result of this section we need some preliminary results which are easy to prove:

Lemma 2. [19] Let $M$ be a DGA-module and $c:\left\{A, M, f^{\prime}, g^{\prime}, \phi^{\prime}\right\}$ be a contraction. If $\phi^{\prime} \mu_{A}\left(g^{\prime} \otimes g^{\prime}\right)=0$, then the morphism $\mu_{M}=f^{\prime} \mu_{A}\left(g^{\prime} \otimes g^{\prime}\right)$ defines a commutative product on $M$. Furthermore, $g^{\prime}$ is a DGA-algebra morphism with regard to the products $\mu_{A}$ and $\mu_{M}$.

Lemma 3. Let $A^{\prime}$ be a DG-algebra, and $c:\left\{A, A^{\prime}, f^{\prime}, g^{\prime}, \phi^{\prime}\right\}$ be a contraction of DG-modules. Then,

$$
\phi^{\prime} \mu_{A}-\mu_{A} \phi^{\prime[\otimes 2]}=\phi^{\prime} \mu_{A} \phi^{\prime[\otimes 2]} d^{[2]}-d \phi^{\prime} \mu_{A} \phi^{\prime[\otimes 2]}-g^{\prime} f^{\prime} \mu_{A} \phi^{\prime[\otimes 2]}
$$

where $\phi^{\prime[\otimes 2]}$ and $d^{[2]}$ denote, respectively, $1_{A} \otimes \phi+\phi \otimes g f$ and $d_{A} \otimes 1_{A}+1_{A} \otimes d_{A}$. Assuming that $f^{\prime}$ is a quasi algebra projection and $\phi^{\prime}$ is a quasi algebra homotopy,

$$
\mu_{A}\left(\phi^{\prime}(a) \otimes \phi^{\prime}(b)\right)=(-1)^{|a|+1} \phi^{\prime}\left(\mu_{A}\left(\phi^{\prime}(a) \otimes b\right)\right)+\phi^{\prime}\left(\mu_{A}\left(a \otimes \phi^{\prime}(b)\right)\right)
$$

where $a, b \in A$.

Lemma 4. The identity $1=s d \phi d+s d g f$ holds on reduced elements with degree greater than zero.

Lemma 5. If $\phi_{n} \mu_{B(A)}(g \otimes g)_{n}\left(\overline{x_{1}} \otimes \overline{x_{2}}\right)=0$, for any a reduced element $\overline{x_{1}} \otimes \overline{x_{2}}$ of degree $n$ then for any element $x_{1} \otimes x_{2}$ of degree $n$ of $X \otimes X$

$$
\left.\phi_{n} \mu_{B(A)}(g \otimes g)_{n}\right)\left(x_{1} \otimes x_{2}\right)=0 .
$$

Now, we state the main result of this section:

Theorem 7. The A-module $(X, d)$ equipped with the morphism $\mu_{X}=f *(g \otimes g)$ becomes a commutative DGA-algebra. Furthermore, $\mathcal{C}$ is an almost-full algebra contraction with regard to the products $\mu_{B(A)}$ and $\mu_{X}$.

PROOF. The proof will be divided into two parts.

Firstly, we show that $\mu_{X}$ defines a commutative product on $X$. To this end, we apply Lemma 2 , in order to prove by induction that $\phi \mu_{B(A)}(g \otimes g)=0$ on reduced elements. Then this relation is extended to $X \otimes X$ by Lemma 5 . We have $\phi_{0}=0$ by construction and for $n>0$, on reduced elements,

$$
\phi_{n} \mu_{B(A)}(g \otimes g)_{n}=-s \phi_{n-1} d_{n} \mu_{B(A)}(g \otimes g)_{n}-s g_{n} f_{n} \mu_{B(A)}(g \otimes g)_{n} .
$$

This equality comes from the fact that $\left.\mu_{B(A)}\right|_{\bar{B}(A) \otimes \bar{B}(A)} \subset \bar{B}(A)$ and the property (7) of $g$. By induction hypothesis

$$
s \phi_{n-1} d_{n} \mu_{B(A)}(g \otimes g)_{n}=s \phi_{n-1} \mu_{B(A)}(g \otimes g)_{n-1} d_{n}^{[2]}=0 .
$$


Now from Lemma 4

$$
\begin{gathered}
s g_{n} f_{n} \mu_{B(A)}(g \otimes g)_{n}=s g_{n} f_{n}\left(s d_{n} \phi_{n-1} d_{n}+s d_{n} g_{n} f_{n}\right) \mu_{B(A)}(g \otimes g)_{n} \\
=s g_{n} f_{n}\left(s d_{n} \phi_{n-1}+s g_{n-1} f_{n-1}\right) \mu_{B(A)}(g \otimes g)_{n-1} d_{n}^{[2]} \\
=s g_{n} f_{n} s d_{n} \phi_{n-1} \mu_{B(A)}(g \otimes g)_{n-1} d_{n}^{[2]}+s g_{n} f_{n} s g_{n-1} f_{n-1} \mu_{B(A)}(g \otimes g)_{n-1} d_{n}^{[2]} .
\end{gathered}
$$

By induction and Lemma 5 the first summand is null. Let us notice that in the second summand $s g_{n-1} f_{n-1} \mu_{B(A)}(g \otimes g)_{n-1}$ can be applied on non-reduced elements, so in order to prove that it is null we need a more sophisticated argument.

We show by induction that if $s g f \mu_{B(A)}(g \otimes g)$ is null on any element of degree less than $n$, then it is possible to extend this property to any element in $X \otimes X$ of degree $n$. The first case of the induction is trivial (for $n=0$ ). We take a generic element $(a \otimes \bar{x}) \otimes\left(a^{\prime} \otimes \bar{x}^{\prime}\right)$ in degree $n$. Thus,

$$
s g_{n} f_{n} \mu_{B(A)}\left(g(a \otimes \bar{x}) \otimes g\left(a^{\prime} \otimes \bar{x}^{\prime}\right)\right)_{n}=(-1)^{\left|a^{\prime}\right||\bar{x}|} s\left(a a^{\prime} g f \mu_{B(A)}\left(g(\bar{x}) \otimes g\left(\bar{x}^{\prime}\right)\right)\right) .
$$

Now, using again Lemma 4

$$
s\left(a a^{\prime} g f \mu_{B(A)}\left(g(\bar{x}) \otimes g\left(\bar{x}^{\prime}\right)\right)\right)=s\left(a a^{\prime} g f(s d \phi d+s d g f) \mu_{B(A)}(g(\bar{x}) \otimes g((\bar{x})))\right),
$$

let us observe that the summands, $(s d \phi d+s d g f) \mu_{B(A)}\left(g(\bar{x}) \otimes g\left(\bar{x}^{\prime}\right)\right)$, are zero by induction hypothesis. This fact completes the proof of the first step.

Secondly, we are proving that $\mathcal{C}$ is an almost-full algebra contraction (i.e., $f$ and $g$ are DGA-algebra morphisms and $\phi$ is a quasi algebra morphism with regard to the products $\mu_{B(A)}$ and $\mu_{X}$ ). Lemma 2 guarantees that $g$ is a DGAalgebra morphism. In order to prove that $f$ is a DGA-algebra morphism we need to see that $\mu_{X}(f \otimes f)=f \mu_{B(A)}$. The proof is by induction on reduced elements and then extended $A$-linearly in each degree. Obviously, we have $\mu_{X}\left(f_{0} \otimes f_{0}\right)=$ $f_{0} \mu_{B(A)}$ and for $n>0$,

$$
\begin{gathered}
\mu_{X}\left(f_{n-i}(\bar{b}) \otimes f_{i}\left(\bar{b}^{\prime}\right)\right)=\mu_{X}\left(t f_{n-i-1} d_{n-i}(\bar{b}) \otimes t f_{i-1} d_{i}\left(\bar{b}^{\prime}\right)\right) \\
=(-1)^{|\bar{b}|} t \mu_{X}\left(t f_{n-i-1} d_{n-i}(\bar{b}) \otimes f_{i-1} d_{i}\left(\bar{b}^{\prime}\right)\right)+t \mu_{X}\left(f_{n-i-1} d_{n-i}(\bar{b}) \otimes t f_{i-1} d_{i}\left(\bar{b}^{\prime}\right)\right) \\
=(-1)^{|\bar{b}|} t \mu_{X}\left(f_{n-i}(\bar{b}) \otimes f_{i-1} d_{i}\left(\bar{b}^{\prime}\right)\right)+t \mu_{X}\left(f_{n-i-1} d_{n-i}(\bar{b}) \otimes f_{i}\left(\bar{b}^{\prime}\right)\right) .
\end{gathered}
$$

In the second identity above we have taken into account that $t$ is a quasi algebra homotopy with respect to the product $\mu_{X}$ and we have applied (12).

On the other hand,

$$
\begin{gathered}
f_{n} \mu_{B(A)}\left(\bar{b} \otimes \bar{b}^{\prime}\right)=t f_{n-1} d_{n} \mu_{B(A)}\left(\bar{b} \otimes \bar{b}^{\prime}\right) \\
=t f_{n-1} \mu_{B(A)}\left(d_{n-i}(\bar{b}) \otimes \bar{b}^{\prime}\right)+(-1)^{|\bar{b}|} t f_{n-1} \mu_{B(A)}\left(\bar{b} \otimes d_{i}\left(\bar{b}^{\prime}\right)\right) \\
=t \mu_{X}\left(f_{n-i-1} d_{n-i}(\bar{b}) \otimes f_{i}\left(\bar{b}^{\prime}\right)\right)+(-1)^{|\bar{b}|} t \mu_{X}\left(f_{n-i}(\bar{b}) \otimes f_{i-1} d_{i}\left(\bar{b}^{\prime}\right)\right),
\end{gathered}
$$

the last identity is obtained from induction hypothesis. So we have actually proved that $f$ is a DGA-algebra morphism. 
Finally, we will prove that $\phi$ is a quasi algebra homotopy, i.e., the conditions

$$
\phi \mu_{B(A)}(\phi \otimes g)=0, \quad \phi \mu_{B(A)}(g \otimes \phi)=0, \quad \phi \mu_{B(A)}(\phi \otimes \phi)=0 \quad \text { hold. }
$$

The proof is by induction. We have $\phi_{0}=0$ by construction, so the above three identities hold. For $n>0$, on reduced elements, the proof consists in replacing $\phi \mu_{B(A)}$ by

$$
-s \phi d \mu_{B(A)}-s g f \mu_{B(A)}=s \phi \mu_{B(A)}(d \otimes 1+1 \otimes d)-s g \mu_{X}(f \otimes f),
$$

then, the summands of the form $s g \mu_{X}(f \otimes f)$ are all null, since $f \phi=0$. Moreover, the summands which contain $d g \otimes \phi$ or $\phi \otimes d g$ are zero, since $d_{n} g_{n}=g_{n-1} d_{n}$. So it is possible to apply induction hypothesis. To sum up, we must only study the summands of the form:

$s \phi \mu_{B(A)}(d \phi \otimes g), \quad s \phi \mu_{B(A)}(g \otimes d \phi), \quad s \phi \mu_{B(A)}(d \phi \otimes \phi), \quad s \phi \mu_{B(A)}(\phi \otimes d \phi)$.

Replacing $d \phi$ by $1-g f-\phi d$ is immediate to see that all summands are null. By $A$-linearity the proof is extended to elements of $B(A)$. This completes the proof.

Finally, we provide the following algorithm for computing (algebra) resolutions which split off of the bar construction of a commutative DGA-algebra $\tilde{A}$, taking as input datum a contractile relatively free resolution of $\Lambda$ over a commutative DGA-algebra $A$, where $A$ and $\tilde{A}$ coincide as graded module.

\section{Algorithm 2. Computing 'algebra' resolutions which split off}

INPUT: A contractile relatively free resolution $(X, t, d)$ of $\Lambda$ over a commutative DG-algebra $A$ where $\left.d \circ t\right|_{\bar{X}}=0$ and $t$ is a quasi algebra homotopy.

A commutative DGA-algebra $\tilde{A}$ which has the same underlying graded $A$-module structure than $A$.

Step 1. Form the canonical comparison contraction $(f, g, \phi): B(A) \Rightarrow X$ using the formulas (5), (4) and (6).

Step 2. Construct the bar constructions $\left(B(\tilde{A}), \partial^{+}\right)$and $(B(A), \partial)$. Define the morphism $\delta=\partial^{+}-\partial$.

Step 3. Perturb the above contraction using $\delta$, (if $\phi \delta$ is nilpotent).

Output: A semi-full algebra contraction $B(\tilde{A}) \Rightarrow \tilde{X}$. Hence, $\tilde{X}$ is an algebra resolution of $\Lambda$ over $\tilde{A}$, where $\mu_{\tilde{X}}=f *(g \otimes g)$.

We point out that the contraction of Step 1 is almost-full (see Theorem 7 ). Furthermore, in the case that $\phi \delta$ is pointwise nilpotent, thus $\delta$ is an algebra perturbation datum of the contraction of Step 1. Hence, using Theorem 3, we conclude with the desired result. The main computational advantage of the algebra structure in $\tilde{X}$ is that it is only necessary to compute the perturbed differential on the generators of $\tilde{X}$ as an algebra, in spite of, on the whole set of generators as a module. We will clarify this aspect in the following example. 


\section{An Example}

Now, we give an example in order to illustrate the main results of the paper. We work with the resolution $Q_{(p)}(w, 2 n) \otimes E(\sigma(w), 2 n+1) \otimes \Gamma\left(\varphi_{p}(w), 2 n p+2\right)$ (see [5). Making use of the main results of the paper we reach the same results on the $A_{\infty}$-structure of this DG-module as Proute in [18. Furthermore, we prove that this complex is a DGA-algebra. Hence, it is an example of a multiplicative $A_{\infty}$-twisted tensor product. Moreover, this resolution can be "perturbed" into a resolution of $\mathbf{Z}_{(p)}$ over $\Gamma(w, 2 n)$. Notice that the way for obtaining the resolution above is different from that given in [2].

Following Cartan's work in 5, we will use in the sequel the suspension ' $\sigma$ ', $p$-transpotence ' $\varphi_{p}$ ' and $k$-th divided power ' $\gamma_{k}$ ' for terming the generators of the DGA-algebras.

Let $p$ be a prime number and $I=\left(w^{p}\right)$ be the ideal generated by $w^{p}$. Then, $Q_{(p)}(w, 2 n)=P(w, 2 n) / I$ is the truncated polynomial algebra on one generator $w$ of degree $2 n$ with zero differential. We consider here the resolution $X=$ $Q_{(p)}(w, 2 n) \otimes E(\sigma(w), 2 n+1) \otimes \Gamma\left(\varphi_{p}(w), 2 n p+2\right)$ where the differential is a derivation and is defined by

$$
d(\sigma(w))=w, \quad d\left(\gamma_{i}\left(\varphi_{p}(w)\right)\right)=w^{p-1} \sigma(w) \gamma_{i-1}\left(\varphi_{p}(w)\right) .
$$

The following degree one morphism $t: X \rightarrow X$ linear over $\Lambda$ (but not over $\left.Q_{(p)}(w, 2 n)\right)$ defined as

$$
t(1)=0, \quad t\left(w^{k} \gamma_{i}\left(\varphi_{p}(w)\right)\right)=w^{k-1} \sigma(w) \gamma_{i}\left(\varphi_{p}(w)\right)
$$

and

$$
t\left(w^{k} \sigma(w) \gamma_{i}\left(\varphi_{p}(w)\right)\right)=\left\{\begin{array}{cc}
\gamma_{i+1}\left(\varphi_{p}(w)\right) & k=p-1 \\
0 & k \neq p-1
\end{array}\right.
$$

is a contracting homotopy for $X$. This explicit formula for $t$ is crucial to many constructions but it is not widely distributed. Moreover, the data set

$$
c_{X}:\{X, \Lambda, \epsilon, \eta, t\}
$$

is a contraction, where $\epsilon_{0}=1_{\Lambda}, \epsilon_{n}=0, n>0$ and $\eta(\lambda)=\lambda \otimes 1 \otimes 1$. Now, by comparison theorem for resolutions and using the formulae (5), (4) and (6) for comparison maps, we have the following $Q_{(p)}(w, 2 n)$-lineal morphisms defined on the reduced complexes by

$$
\begin{gathered}
f\left[w^{r_{1}}\left|w^{t_{1}}\right| \ldots\left|w^{r_{m}}\right| w^{t_{m}}\right]=\left\{\prod_{k=1}^{n} \delta_{p, r_{k}+t_{k}}\right\} \gamma_{m}\left(\varphi_{p}(w)\right), \\
f\left[w^{l}\left|w^{r_{1}}\right| w^{t_{1}}|\ldots| w^{r_{m}} \mid w^{t_{m}}\right]=w^{l-1}\left\{\prod_{k=1}^{n} \delta_{p, r_{k}+t_{k}}\right\} \sigma(w) \gamma_{m}\left(\varphi_{p}(w)\right),
\end{gathered}
$$

where the symbols $\delta_{i, j}$ are defined by: $\delta_{i, j}= \begin{cases}1 & i=j, \\ 0 & i \neq j\end{cases}$

$$
g(\sigma(w))=[w], \quad g\left(\gamma_{i}\left(\varphi_{p}(w)\right)\right)=\left[w^{p-1}|w| \stackrel{i \text {-times }}{\cdots}\left|w^{p-1}\right| w\right],
$$




$$
g\left(\sigma(w) \gamma_{i}\left(\varphi_{p}(w)\right)\right)=\left[w\left|w^{p-1}\right| \stackrel{i \text {-times }}{\cdots}|w| w^{p-1} \mid w\right]
$$

and the homotopy operator $\phi$ is defined by $-\psi$, where

$$
\begin{aligned}
& \psi[]=0, \quad \psi[w]=0, \\
& \psi\left[w^{x}\right]=\left[w^{x-1} \mid w\right], \quad 1<x<p \\
& \psi\left[w^{x} \mid w^{y}\right]=\left[w^{x}\left|w^{y-1}\right| w\right], \\
& \psi\left[z\left|w^{x}\right| w^{y}\right]=\left[z\left|w^{x}\right| w^{y-1} \mid w\right]+\delta_{p, x+y}\left[\psi(z)\left|w^{p-1}\right| w\right]
\end{aligned}
$$

for $z \in \bar{B}\left(Q_{(p)}(w, 2 n)\right)$.

It is a straightforward computation to verify that $\left.d t\right|_{\bar{x}}=0$. Then by Theorem 1 we can state that the data set

$$
\mathcal{C}_{B-X}:\left\{B\left(Q_{(p)}(w, 2 n)\right), X, f, g, \phi\right\}
$$

is a contraction: the canonical comparison contraction between $B\left(Q_{(p)}(w, 2 n)\right)$ and $X$. It is immediate to see that $t$ is a quasi algebra homotopy, then by Theorem 7 we can guarantee that $\mathcal{C}_{B-X}$ is an almost-full contraction.

Now, we can apply Algorithm 1 to the resolution $X$, and define the degree minus one morphism $\tau: E(\sigma(w), 2 n+1) \otimes \Gamma\left(\varphi_{p}(w), 2 n p+2\right) \rightarrow Q_{(p)}(w, 2 n)$ by

$$
\tau(\sigma(w))=\theta g(\sigma(w))=w \quad \text { and } \quad \tau=0 \quad \text { otherwise }
$$

which is a $A_{\infty}$-twisting cochain.

Working with coefficients in $\mathbf{Z}_{p}$, Proute determined in [18] the $A_{\infty}$-coalgebra structure of $E(\sigma(w), 2 n+1) \otimes \Gamma\left(\varphi_{p}(w), 2 n p+2\right)$ given the following formulae:

$\Delta_{h}=0, \quad h \neq 2, p$,

$$
\begin{aligned}
& \Delta_{2}\left(\sigma(w)^{j} \gamma_{i}\left(\varphi_{p}(w)\right)\right)=\sum_{k=0}^{j} \sum_{l=0}^{i} \sigma(w)^{k} \gamma_{l}\left(\varphi_{p}(w)\right) \otimes \sigma(w)^{j-k} \gamma_{i-l}\left(\varphi_{p}(w)\right), \\
& \Delta_{p}\left(\sigma(w)^{j} \gamma_{i}\left(\varphi_{p}(w)\right)\right)=\sum_{l_{1}+\cdots+l_{p}=i-1} \sigma(w)^{j+1} \gamma_{l_{1}}\left(\varphi_{p}(w)\right) \otimes \cdots \otimes \sigma(w)^{j+1} \gamma_{l_{p}}\left(\varphi_{p}(w)\right) .
\end{aligned}
$$

In [18, pp.148-149] it is proved that

$$
d=(\mu \otimes 1)(1 \otimes \tau \otimes 1)\left(1 \otimes \Delta_{2}\right)+\left(\mu^{(p)} \otimes 1\right)\left(1 \otimes \tau^{\otimes i-1} \otimes 1\right)\left(1 \otimes \Delta_{p}\right)
$$

as the output of the Algorithm 1 states.

Summing up, $X=Q_{(p)}(w, 2 n) \otimes_{\tau} E(\sigma(w), 2 n+1) \otimes \Gamma\left(\varphi_{p}(w), 2 n p+2\right)$ is a multiplicative $A_{\infty}$-twisted tensor product, i.e., $X$ is a DGA-algebra and an $A_{\infty}$-TTP simultaneously.

For the remainder of this example we have taken $\mathbf{Z}_{(p)}$ ( $\mathbf{Z}$ localized at prime $p)$ as the ground ring. In the following, we give the outline of a process for 
constructing a resolution of $\mathbf{Z}_{(p)}$ over $\Gamma(w, 2 n)$. It is obtained by perturbing a resolution of $\mathbf{Z}_{(p)}$ over $\otimes_{i \geq 0} Q_{(p)}\left(w_{i}, 2 n p^{i}\right)$.

Firstly, we use an isomorphism of DGA-algebras [19, Prop. 5.24] between $\Gamma(w, 2 n)$ and $\tilde{\otimes}_{i \geq 0} Q_{(p)}\left(w_{i}, 2 n p^{i}\right)$. As $\mathbf{Z}_{(p)}$-module, this last DGA-algebra is equal to the ordinary tensor product $\otimes_{i \geq 0} Q_{(p)}\left(w_{i}, 2 n p^{i}\right)$. Its multiplicative law is given by

$$
w_{i}^{k} w_{j}^{h}= \begin{cases}w_{i}^{k} \otimes w_{j}^{h} & \text { if } i \neq j, \\ w_{i}^{k+h} & \text { if } i=j \text { and } h+k<p, \\ -p w_{i}^{t} w_{i+1} & \text { if } i=j \text { and } h+k=p+t\end{cases}
$$

From now on, we will identify the generators $w_{i}$ of the truncated algebras with the elements $\gamma_{p^{i}}(w)$ of $\Gamma(w, 2 n)$; in fact, the image by the isomorphism of $w_{i}$ coincides with $\gamma_{p^{i}}(w)$ excluding the coefficient.

Secondly, we give an explicit contracting homotopy $t^{\otimes}$ for the resolution

$$
X^{\otimes}=\otimes_{i \geq 0} Q_{(p)}\left(w_{i}, 2 n p^{i}\right) \otimes\left(\otimes_{i \geq 0} E\left(\sigma\left(w_{i}\right), 2 n p^{i}+1\right) \otimes \Gamma\left(\varphi_{p}\left(w_{i}\right), 2 n p^{i}+2\right)\right) .
$$

To this end, we use that the complex above is just the tensor product complex

$$
\otimes_{i \geq 0}\left(Q_{(p)}\left(w_{i}, 2 n p^{i}\right) \otimes E\left(\sigma\left(w_{i}\right), 2 n p^{i}+1\right) \otimes \Gamma\left(\varphi_{p}\left(w_{i}\right), 2 n p^{i}+2\right)\right)
$$

and the formula for $t^{\otimes}$ is:

$$
t \otimes 1 \otimes 1 \otimes \ldots+\eta \epsilon \otimes t \otimes 1 \otimes 1 \ldots+\eta \epsilon \otimes \eta \epsilon \otimes t \otimes 1 \otimes 1 \otimes \ldots+\cdots,
$$

thus,

$$
t^{\otimes}\left(1 \otimes{ }^{l \text {-times }} \otimes 1 \otimes x \otimes z\right)=1 \otimes{ }^{l \text {-times }} \otimes 1 \otimes t(x) \otimes z
$$

where $|x|>0, x \in Q_{(p)}\left(w_{l}, 2 n p^{l}\right) \otimes E\left(\sigma\left(w_{l}\right), 2 n p^{l}+1\right) \otimes \Gamma\left(\varphi_{p}\left(w_{l}\right), 2 n p^{l}+2\right)$ and $z \in \otimes_{i>l}\left(Q_{(p)}\left(w_{i}, 2 n p^{i}\right) \otimes E\left(\sigma\left(w_{i}\right), 2 n p^{i}+1\right) \otimes \Gamma\left(\varphi_{p}\left(w_{i}\right), 2 n p^{i}+2\right)\right)$.

Since the contraction (13) is an almost-full algebra contraction, and the class of almost-full contraction is closed by tensor product [19, it follows that $t^{\otimes}$ is a quasi algebra homotopy.

Now, by comparison theorem for resolutions and using the formulae (5), (4) and (6) for comparison maps, it is possible to construct three morphisms denoted by $f^{\otimes}, g^{\otimes}, \phi^{\otimes}$.

Since $\left.t\right|_{\bar{X}}=0$, we have that $\left.d t^{\otimes}\right|_{X^{\otimes}}=0$. Then by Theorem 1 we can state that the data set

$$
\left\{B\left(\otimes_{i \geq 0} Q_{(p)}\left(w_{i}, 2 n p^{i}\right)\right), X^{\otimes}, f^{\otimes}, g^{\otimes}, \phi^{\otimes}\right\}
$$

is a contraction. Besides, it is an almost-full algebra contraction thanks to Theorem[7. Hence, in particular, $g^{\otimes}$ is DGA-algebra morphism, then $g^{\otimes}$ is completely determined by $g$, i.e.,

$$
g^{\otimes}\left(x_{0} \otimes \cdots \otimes x_{n}\right)=g\left(x_{0}\right) * g\left(x_{1}\right) * \cdots * g\left(x_{n}\right), \forall n \geq 0 ;
$$

where $x_{i} \in E\left(\sigma\left(w_{i}\right), 2 n p^{i}+1\right) \otimes \Gamma\left(\varphi_{p}\left(w_{i}\right), 2 n p^{i}+2\right)$ and $*$ denotes the well-known shuffle product in the bar construction. 
The projection $f^{\otimes}$ is given by

$$
\begin{aligned}
& f^{\otimes}\left[w_{i_{1}}^{k_{1}} \otimes z_{1}\left|w_{i_{2}}^{k_{2}} \otimes z_{2}\right| \ldots \mid w_{i_{n}}^{k_{n}} \otimes z_{n}\right] \\
& = \begin{cases}w_{i_{1}}^{k_{1}-1} z_{1} w_{i_{2}}^{k_{2}-1} z_{2} \cdots w_{i_{n}}^{k_{n}-1} z_{n} \sigma\left(w_{i_{1}}\right) \sigma\left(w_{i_{2}}\right) \cdots \sigma\left(w_{i_{n}}\right) & i_{1}<i_{2}<\cdots<i_{n}, \\
\left\{\prod_{j=0}^{m-1} \delta_{p, k_{2 j+1}+k_{2 j+2}}\right\}^{2} z_{1} \cdots z_{n} \gamma_{m}\left(\varphi_{p}\left(w_{i_{1}}\right)\right) & i_{1}=i_{2}=\cdots=i_{n=2 m}, \\
\left.\prod_{j=1}^{m} \delta_{p, k_{2 j}+k_{2 j+1}}\right\} w_{i_{1}}^{k_{1}-1} z_{1} \cdots z_{n} \sigma\left(w_{i_{1}}\right) \gamma_{m}\left(\varphi_{p}\left(w_{i_{1}}\right)\right) & i_{1}=i_{2}=\cdots=i_{n=2 m+1}, \\
f^{\otimes}\left[a_{i_{1}}|\ldots| a_{i_{1}}\right] \otimes \cdots \otimes f^{\otimes}\left[a_{i_{l_{h}}}|\ldots| a_{i_{n}}\right] & i_{1}=\ldots=i_{l_{1}}<\cdots<i_{l_{h}}=\ldots=i_{n} \\
0 & \text { otherwise, }\end{cases}
\end{aligned}
$$

where $z_{j} \in \otimes_{i>j} Q_{(p)}\left(w_{i}, 2 n p^{i}\right)$ and $a_{j}=w_{i_{j}}^{k_{j}} \otimes z_{j}$. And the homotopy operator $\phi^{\otimes}$ is defined by $-\psi^{\otimes}$,

$$
\begin{aligned}
& \psi^{\otimes}[]=0, \\
& \psi^{\otimes}\left[w_{i_{1}}^{k_{1}} \otimes z_{1}\right]=\left[w_{i_{1}}^{k_{1}-1} z_{1} \mid w_{i_{1}}\right] \\
& \psi^{\otimes}\left[w_{i_{1}}^{k_{1}} z_{1} \mid w_{i_{2}}^{k_{2}} z_{2}\right]= \begin{cases}{\left[w_{i_{1}}^{k_{1}} z_{1}\left|w_{i_{2}}^{k_{2}-1} z_{2}\right| w_{i_{2}}\right]+\left[w_{i_{1}}^{k_{1}-1} z_{1} w_{i_{2}}^{k_{2}-1} z_{2}\left|w_{i_{1}}\right| w_{i_{2}}\right]-} & i_{1}<i_{2}, \\
{\left[w_{i_{1}}^{k_{1}-1} z_{1} w_{i_{2}}^{k_{2}-1} z_{2}\left|w_{i_{2}}\right| w_{i_{1}}\right]} & i_{1} \geq i_{2} . \\
{\left[w_{i_{1}}^{k_{1}} z_{1}\left|w_{i_{2}}^{k_{2}-1} z_{2}\right| w_{i_{2}}\right]} & \end{cases}
\end{aligned}
$$

The situation in higher degrees is similar but slightly more complicated and is left to the interested reader.

For the sake of clarity, we will write the DGA-algebras without denoting the degree of the generators.

[19, Prop. 5.24] tells that there is an isomorphism between $\Gamma(w)$ and a tensor product $\tilde{\otimes}_{i \geq 0} Q_{(p)}\left(\gamma_{p^{i}}(w)\right)$.

$\left.\tilde{\otimes}_{i \geq 0} Q_{(p)}\left(\gamma_{p^{i}}(w)\right)\right)$ and $\left.\otimes_{i \geq 0} Q_{(p)}\left(\gamma_{p^{i}}(w)\right)\right)$ have the same underlying graded $\mathbf{Z}_{(p)}$-module structure and because of this, $\left.B\left(\tilde{\otimes}_{i \geq 0} Q_{(p)}\left(\gamma_{p^{i}}(w)\right)\right)\right)$ has the same underlying graded $\mathbf{Z}_{(p)}$-module structure as $\left.B\left(\otimes_{i \geq 0} Q_{(p)}\left(\gamma_{p^{i}}(w)\right)\right)\right)$. Let $B$ denote this graded $\mathbf{Z}_{(p)}$-module structure for either case. Thus $B$ supports two different differentials, viz., the bar construction differential $\partial^{+}$for $\left.\tilde{\otimes}_{i \geq 0} Q_{(p)}\left(\gamma_{p^{i}}(w)\right)\right)$ and the bar construction differential $\partial$ for $\left.\otimes_{i \geq 0} Q_{(p)}\left(\gamma_{p^{i}}(w)\right)\right)$. Let $\delta=\partial^{+}-\partial$ be the perturbation of the DG-module $B$. The formula for $\delta$, up to sign, is

$$
\begin{aligned}
& \delta\left(\otimes_{i \geq 0}\left(\gamma_{p^{i}}(w)\right)^{h_{0, i}}\left[\otimes_{i \geq 0}\left(\gamma_{p^{i}}(w)\right)^{h_{1, i}}\left|\otimes_{i \geq 0}\left(\gamma_{p^{i}}(u)\right)^{h_{2, i}}\right| \ldots\right]\right) \\
& =\cdots p\left(\gamma_{p^{l}}(w)\right)^{t} \gamma_{p^{l+1}}(w) \cdots\left[\otimes_{i \geq 0}\left(\gamma_{p^{i}}(w)\right)^{h_{2, i}} \mid \ldots\right] \\
& \quad+\otimes_{i \geq 0}\left(\gamma_{p^{i}}(w)\right)^{h_{0, i}}\left[\cdots p\left(\gamma_{p^{j}}(w)\right)^{t} \gamma_{p^{j+1}}(w) \cdots \mid \ldots\right]+\ldots
\end{aligned}
$$

The first summand appears (it is non-zero) if there exists at least one value for $i$ such that $h_{0, i}+h_{1, i}=p+t$ where $0 \leq t \leq p-2$. The second summand 
appears if there exists at least one value for $i$ such that $h_{1, i}+h_{2, i}=p+t$ where $0 \leq t \leq p-2$. And so on.

It is clear that $\delta$ is a derivation and represents the perturbation induced in the differential of $\left.B\left(\otimes_{i \geq 0} Q_{(p)}\left(\gamma_{p^{i}}(w)\right)\right)\right)$ by the modification produced in the product of the algebra $\left.\otimes_{i \geq 0} Q_{(p)}\left(\gamma_{p^{i}}(w)\right)\right)$. In this situation, there is a formal process (the Basic Perturbation Lemma) which, taking as the input data the contraction (14) and the perturbation $\delta$, when $\delta \phi^{\otimes}$ is nilpotent in each degree, it gives a new contraction

$$
\begin{gathered}
B\left(\tilde{\otimes}_{i \geq 0} Q_{(p)}\left(\gamma_{p^{i}}(w)\right)\right) \\
\Downarrow \\
\left(\otimes_{i \geq 0} Q_{(p)}\left(\gamma_{p^{i}}(w)\right) \otimes\left(\otimes_{i \geq 0} E\left(\sigma \gamma_{p^{i}}(w)\right) \otimes \Gamma\left(\varphi_{p} \gamma_{p^{i}}(w)\right)\right), d+d_{\delta}\right)
\end{gathered}
$$

Our aim here is to verify that $\delta \phi^{\otimes}$ is nilpotent in each degree. To this end, we take an element of the form

$$
\otimes_{i=0}^{n}\left(\gamma_{p^{i}}(w)\right)^{h_{0, i}}\left[\otimes_{i=0}^{n}\left(\gamma_{p^{i}}(w)\right)^{h_{1, i}}|\ldots| \otimes_{i=0}^{n}\left(\gamma_{p^{i}}(u)\right)^{h_{l, i}}\right] \in B,
$$

the number: $\sum_{j=0}^{l} \sum_{i=0}^{n} h_{j, i}$ defines a filtration in $B$.

It is easy to see that $\phi^{\otimes}$ does not increase the filtration degree. On the other hand, $\delta$ either lowers the filtration degree or is null, $\forall n, \forall l \in \mathbf{N}$. Then, $\delta \phi^{\otimes}$ either lowers filtration or is null, and this means that this composition is nilpotent in each degree.

Taking into account Theorem 3, the contraction above is a semi-full algebra contraction. Notice that the product on the second complex coincides with the product on $X^{\otimes}$. Then, we only need to compute $d_{\delta}$ on the algebra generators, in order to compute $d_{\delta}$ on all module generators. Moreover, we shall show that

$$
\phi^{\otimes} \delta g^{\otimes}=0
$$

Let us observe that $g^{\otimes}$ carries any algebra generator $x$ of the reduced complex into an element $y$ of the form $\left[\gamma_{p^{i}}(w)\right]$ or $\left[\left(\gamma_{p^{i}}(w)\right)^{p-1} \mid \gamma_{p^{i}}(w)\right]$. Now, we study the image of $y$ under $\delta$. It is not difficult to see that this image is zero if $y=\left[\gamma_{p^{i}}(w)\right]$ and $p\left[\gamma_{p^{i+1}}(w)\right]$ if $y=\left[\left(\gamma_{p^{i}}(w)\right)^{p-1} \mid \gamma_{p^{i}}(w)\right]$. Since $\phi^{\otimes}\left[\gamma_{p^{i+1}}(w)\right]=0$, we obtain the desired result.

Consequences of (16) are:

$$
\left(g^{\otimes}\right)_{\delta}=g^{\otimes}, \quad d_{\delta}=f^{\otimes} \delta g^{\otimes} .
$$

Summing up, (15) is a resolution of $\mathbf{Z}_{p}$ over $\Gamma(w)$ where

$$
\begin{aligned}
d_{\delta}\left(\sigma \gamma_{p^{i}}(w)\right) & =f^{\otimes} \delta g^{\otimes}\left(\sigma \gamma_{p^{i}}(w)\right)=f^{\otimes} \delta\left[\gamma_{p^{i}}(w)\right]=f^{\otimes}(0)=0, \\
d_{\delta}\left(\varphi_{p} \gamma_{p^{i}}(w)\right) & =f^{\otimes} \delta g^{\otimes}\left(\varphi_{p} \gamma_{p^{i}}(w)\right)=f^{\otimes} \delta\left[\left(\gamma_{p^{i}}(w)\right)^{p-1} \mid \gamma_{p^{i}}(w)\right] \\
& =f^{\otimes}\left(p\left[\gamma_{p^{i+1}}(w)\right]\right)=p \sigma \gamma_{p^{i+1}}, \\
d_{\delta}\left(\gamma_{k} \varphi_{p} \gamma_{p^{i}}(w)\right) & =f^{\otimes} \delta g^{\otimes}\left(\gamma_{k} \varphi_{p} \gamma_{p^{i}}(w)\right)=p \sigma \gamma_{p^{i+1}}(w) \gamma_{k-1} \varphi_{p} \gamma_{p^{i}}(w) .
\end{aligned}
$$




\section{References}

1. Álvarez, V., Armario, J.A., Frau, M.D., Real, P.: Transferring TTP-structures via contraction. Homology, Homotopy Appl. 7 (2) (2005) 41-54

2. Armario, J.A., Real, P., Silva, B.: On $p$-minimal homological models of twisted tensor products of elementary complexes localized over a prime. in: J. McCleary (ed.), Higher Homotopy Structures in Topology and Mathematical Physics (Poughkeepsie, NY, 1996), Contemp. Math.,A.M.S, Providence, RI, 1999, pp. 303-314

3. Barnes, D. W., Lambe, L. A.: A fixed point approach to homological perturbation theory. Proc. Amer. Math. Soc. 112 (3) (1991) 881-892

4. Brown, R.: The twisted Eilenberg-Zilber theorem. Celebrazioni Archimedee del secolo XX, Simposio di topologia (1967) 34-37

5. Cartan, H.: Algèbres d'Eilenberg-Mac Lane. Séminaire H. Cartan 1954/55, (exposé 2 à 11). Ecole Normale Supérieure, Paris, (1956)

6. Eilenberg, S., Mac Lane, S.: On the groups $H(\pi, n)$, I. Annals of Math. 58 (1953) $55-106$

7. Gugenheim, V.K.A.M., Lambe, L.A.: Perturbation theory in Differential Homological Algebra I. Illinois J. Math. 33 (4) (1989) 566-582

8. Gugenheim, V.K.A.M., Lambe, L.A., Stasheff, J.D.: Perturbation theory in Differential Homological Algebra II. Illinois J. Math. 35 (3) (1991) 357-373

9. Huebschmann, J., Kadeishvili, T.: Small models for chain algebras. Math. Z. 209 (1991) 245-280

10. Johansson, L., Lambe, L.A.: Transferring algebra structures up to homology equivalence. Math. Scan. 88 (2) (2001) 181-200

11. Johansson, L., Lambe, L.A., Sköldberg, E.: On constucting resolutions over the polynomial algebra Homology, Homotopy Appl., 4 (2) (2002) 315-336

12. Lambe, L.A.: Resolutions via homological perturbation. J. Symbolic Comp. 12 (1991) $71-87$

13. Lambe, L.A.: Homological perturbation theory. Hochschild homology and formal groups. In: Proc. Conference on Deformation Theory and Quantization with Applications to Physics, (Amherst, MA, June 1990), American Mathematical Society, Providence, RI, 1992, 183-218

14. Lambe, L.A.: Resolutions which split off of the bar construction. J. Pure Appl. Algebra, 84 (1993) 311-329

15. Lambe, L.A., Stasheff, J.D.: Applications of perturbation theory to iterated fibrations. Manuscripta Math. 58 (1987) 367-376

16. Mac Lane, S.: Homology. Classics in Mathematics Springer-Verlag, Berlin, (1995). Reprint of the 1975 edition.

17. May, J.P.: The cohomology of restricted Lie algebras and of Hopf algebras. J. Algebra, 3 (1966) 123-146

18. Prouté, A.: Algèbres différentielles fortement homotopiquement associatives $\left(A_{\infty}\right.$ algèbre). Ph. D. Thesis, Université Paris VII (1984)

19. Real, P.: Homological Perturbation Theory and Associativity. Homology, Homotopy Appli. 2 (2000) 51-88

20. Stasheff, J.D.: Homotopy Associativity of $H$-spaces I, II. Trans. A.M.S, 108 (1963) $275-312$ 